\title{
Increased $\gamma / \delta$-positive T-cells in blood and bronchoalveolar lavage of patients with sarcoidosis and hypersensitivity pneumonitis
}

\author{
M. Raulf*, V. Liebers*, C. Steppert**, X. Baur*
}

Increased $\gamma / \delta$-positive T-cells in blood and bronchoalveolar lavage of patients with sarcoidosis and hypersensitivity pneumonitis. M. Raulf, V. Liebers, C. Steppert, X. Baur. CERS Journals Ltd 1994.

ABSTRACT: A small population of T-cells does not express the conventional Tcell receptor (TCR), characterized by the $\alpha$ and $\beta$ polypeptide chains $(\alpha / \beta$ TCR) but two polypeptides termed $\gamma$ and $\delta(\gamma / \delta$ TCR). Changes in $\gamma / \delta$ TCR expression may be relevant as the cause or consequence of several diseases. Our study was undertaken to determine and compare the distribution of T-cells expressing $\gamma / \delta$ TCR in blood and bronchoalveolar lavage (BAL) of patients with sarcoidosis, hypersensitivity pneumonitis (HP), idiopathic pulmonary fibrosis (IPF), and of healthy controls. In addition, the association between $\gamma / \delta$ TCR of blood T-lymphocytes and accessory molecules was analysed.

Using direct immunofluorescence with the anti- $\gamma / \delta$ TCR and anti-CD3 monoclonal antibodies (MoAbs) followed by flow cytometric analysis, the blood of patients with pulmonary sarcoidosis, HP, IPF and of healthy controls was analysed. To reveal the association between $\gamma / \delta$ TCR of blood T-lymphocytes and the accessory molecules, expression of CD4, CD8 and CD25 were determined.

Calculating the percentage and the total number of CD3 $+\gamma / \delta$ TCR cells in blood, the data indicated a significant increase of $\gamma / \delta \mathrm{T}$-cells in individuals with pulmonary sarcoidosis and HP, compared to healthy controls and IPF patients. In sarcoidosis patients with elevated CD3 $+\gamma / \delta$ TCR levels, significantly lower CD4/CD8 ratios were observed. In addition, our data demonstrate a correlation between the decrease of CD4+ cells in blood and the amplified appearance of $\gamma / \delta$ TCR expression in sarcoidosis patients, but not in HP patients. $\gamma / \delta$ TCR + cells were either CD8-positive (approximately 50\%), or had a "double-negative phenotype" (CD4-/CD8-) (remaining 50\%). Circulating blood $\gamma / \delta$ T-cells were CD25-negative, suggesting a lack of activation. The total number of CD3+ $\gamma / \delta$ TCR+-bearing lymphocytes in BAL fluid was increased in sarcoidosis and HP, compared with IPF. A corresponding increase of CD3 $+\gamma / \delta$ TCR + cells was observed in blood and BAL of sarcoidosis patients.

In conclusion, our data indicate that levels of $\gamma / \delta \mathrm{T}$-cells are increased in blood and BAL fluid of patients with sarcoidosis and HP. Although this suggests a pathogenetic role for $\gamma / \delta$ TCR, it is not clear whether its expression is a causal factor, or a consequence, of these diseases.

Eur Respir J., 1994, 7, 140-147.
*Berufsgenossenschaftliches Forschungsinstitut für Arbeitsmedizin (BGFA), Institut an der Ruhr-Universität Bochum, Bochum, Germany. **Bezirksklinikum Kutzenberg, Klinik für Erkrankungen der Atmungsorgane, Ebensfeld, Germany.

Correspondence: M. Raulf

Berufsgenossenschaftliches Forschungsinstitut für Arbeitsmedizin (BGFA)

Abt. Immunologie

Gilsingstr. 14

W-44789 Bochum

Germany

Keywords: Accessory molecules

bronchoalveolar lavage

flow cytometric analysis

hypersensitivity pneumonitis sarcoidosis

T-cell receptor expression

Received: August 61992

Accepted after revision July 261993
The T-cell antigen receptor (TCR), a heterodimer closely associated with the CD3 complex on cell surfaces, and composed of $\alpha$ - and $\beta$-chains, is expressed on most Tcells. Normally, antigens are recognized by $\alpha / \beta$ T-cells, as short peptides presented by major histocompatibility complex (MHC) molecules. Another type of recognition occurs with bacterial superantigens, which bind to monomorphic regions of class II MHC molecules [1]. These superantigens activate $\alpha / \beta T$-cells, which express particular $\mathrm{V} \beta$ gene products, irrespective of their nominal antigenic specificity in an MHC-unrestricted manner. A minor population of T-cells, bearing an antigen receptor which is composed of $\gamma$ and $\delta$ subunits was recently identified [2]. The nature of the antigens recognized by $\gamma / \delta \mathrm{T}-$ cells, and the mode of their presentation, are still largely unknown. A subgroup of $\gamma / \delta \mathrm{T}$-cells, ex-pressing TCR by using $\mathrm{V} \gamma 9$ and $\mathrm{V} \delta 2$ gene product pairs, responds to mycobacterial antigens, representing a superantigenspecific phenomenon [3]. Changes in $\gamma / \delta$ TCR expression may be relevant as the cause or consequence of several diseases. $\gamma / \delta \mathrm{T}$-cells are increased in some infectious diseases, e.g. localized cutaneous leishmaniasis, reversal 
reactions in leprosy, the area surrounding zones of necrosis in tuberculous lymphadenitis, and the peripheral blood of patients with measles, and in immunological disorders such as rheumatoid arthritis and coeliac disease [2].

Sarcoidosis is a chronic, nonmalignant, multisystem disease, often affecting the lungs. The appearance of $\mathrm{T}$ lymphocytes, especially CD4-positive T-lymphocytes, can be demonstrated in increased numbers by bronchoalveolar lavage (BAL) [4]. Studies to understand the pathogenesis of this disease have focused on evaluating the T-cell activation, as a result of antigen interaction with the TCR [5]. Increased numbers of $\gamma / \delta T$-lymphocytes in BAL and blood have been reported in a subgroup of patients with sarcoidosis [6].

Hypersensitivity pneumonitis (HP), also called extrinsic allergic alveolitis (EAA), is a chronic inflammatory lung disease [7, 8], due to a wide variety of environmentally and occupationally encountered organic dusts (e.g. spores from bacteria or saprophytic fungi, bird antigens), and some chemicals (e.g. isocyanates). Recent studies have reported that HP was associated with an influx of CD8+ T-lymphocytes with cytotoxic/suppressor function and macrophage activation in the BAL [9, 10]. The reason why HP only develops in some exposed persons is unknown. However, whilst specific antibodies occur in the blood of both exposed asymptomatic persons and in affected patients, specifically sensitized T-lymphocytes seem to occur more frequently in patients. This finding suggests that T-cell mediated, delayed hypersensitivity mechanisms are involved in the pathogenesis [11].

Sarcoidosis and HP are two different interstitial lung disorders, with specific pathogenetic mechanisms and characteristics. Little is known about similarities between these two diseases concerning the distribution of the $\gamma / \delta$ TCR lymphocytes in peripheral blood and BAL and their association to various cell surface molecules. Therefore, the present study was undertaken to determine and compare the distribution of T-cells expressing $\gamma / \delta$ TCR in blood and BAL of patients with sarcoidosis, HP and idiopathic pulmonary fibrosis (IPF) and healthy controls, applying the method of immunophenotyping by flow cytometry. Furthermore, T-cells of the above-mentioned patient groups were analysed to correlate the different T-cell subsets with the $\gamma / \delta \mathrm{TCR}$ expression.

\section{Materials and methods}

\section{Study population}

All patients were diagnosed on the basis of conventional clinical criteria and results of transbronchial lung biopsy.

Sarcoidosis. The group of sarcoidosis patients included 31 individuals, 11 males and 20 females, aged 20-55 yrs. Six of them were current smokers and seven were ex-smokers. Eleven sarcoidosis patients had enlarged hilar and/or mediastinal lymph nodes (stage I), and 20 combined enlarged thoracic lymph nodes and parenchymal lung infiltrates (stage II). Bronchoalveolar lavage was performed in 20 of the 31 patients.

Hypersensitivity pneumonitis (HP). Thirteen individuals with HP were examined, 3 females and 10 males, aged 31-65 yrs. Two were smokers and three were exsmokers. Diagnoses were based on typical case histories, the presence of antigen-specific antibodies, an abnormal chest X-ray and/or a restrictive ventilation pattern $[12,13]$. This group, including patients with farmers' lung disease $(n=6)$, isocyanate alveolitis $(n=1)$, and bird/pigeon fanciers' lung $(n=6)$, was characterized by the typical symptoms mentioned above and in BAL by a CD4/CD8 ratio below 1.0, increased numbers of activated T-cells (CD3/human leucocyte antigen-DR (HLADR), CD25) and natural killer cells (CD57+/CD56+). In five of the patients, BAL was performed $20 \mathrm{~h}$ after antigen provocation, and showed neutrophils compatible with an acute form of HP.

Idiopathic pulmonary fibrosis (IPF). Twelve individuals with IPF, 10 males and 2 females, aged 43-78 yrs (mean age $57 \pm 14 \mathrm{yrs}$ ) were investigated. Four were smokers and one was an ex-smoker. There were no clinical signs of HP and no histories of occupational or private exposure to organic or inorganic dusts in this group. The patients were without evidence of collagen vascular disease. In this group, BAL lymphocytes were not elevated, and the CD4/CD8 ratio in the BAL was within the normal range (1.1-3.5). A characteristic feature of BAL was an increased percentage of granulocytes (neutrophils and/or eosinophils) up to $15 \%$.

Control group. The control group consisted of 21 healthy volunteers, 12 males and 9 females, aged 22-56 yrs. Seven were smokers and two were ex-smokers. This group was only investigated for blood studies; a BAL was not performed.

\section{$B A L$ procedure and $B A L$ cell preparation}

BAL was performed using $100 \mathrm{ml}$ sterile saline solution. The percentage return of lavage fluid was determined for each BAL, and ranged between 48-85 ml $(60 \pm 11 \mathrm{ml}, \mathrm{n}=32$; mean $\pm \mathrm{SD})$. The lavage fluid was filtered through a single layer of coarse gauze and centrifuged $\left(200 \times \mathrm{g}, 10 \mathrm{~min} 4^{\circ} \mathrm{C}\right)$ to sediment cellular material for cytological and flow cytometric examinations. A trypan blue exclusion test for cell viability was performed, indicating that more than $95 \%$ of cells were viable. The number of cells presented in BAL was determined, and an aliquot of the cell suspension was used to prepare cytocentrifuge slides (Cytospin 3, Shandon, Frankfurt, Germany). The slides were stained using May-Grünwald/Giemsa, and a differential cell count was performed by examination of 300 nonepithelial cells. 


\section{Monoclonal antibodies (MoAbs)}

The following conjugated MoAbs were used to detect lymphocytes, T-cells and T-cell subpopulation: fluorescein isothiocyanate (FITC)-conjugated anti-CD45 (pan leucocyte antigen), phycoerythrin (PE)-conjugated anti-CD8 (leu-2a, recognizing CD8+ "cytotoxic/suppressor" T-cells), PE-conjugated anti-CD4 (leu-3a, recognizing CD4+ "helper/inducer" T-lymphocytes), PE-conjugated anti-CD3 (leu-4, recognizing all CD3+ T-lymphocytes), PE-/or FITC-conjugated anti-CD25 (recognizing the interleukin-2 (IL-2) receptor), FITC-anti-CD4/PE-anti-CD8 (leu-3a/leu-2a) and FITC-anti-CD45/PE-anti-CD14 (monocyte specific). To determine the TCR phenotype of T-lymphocytes, anti-TCR $\alpha / \beta-1$ (identifying all $\alpha / \beta$ T-lymphocytes) and anti-TCR $\gamma / \delta$ (recognizing all $\gamma / \delta$ T-lymphocytes) were used in the fluorescein (FITC)conjugated form. Control antibodies included isotypematched PE-conjugated and FITC-conjugated mouse antibodies. The MoAbs were purchased from BectonDickinson, Heidelberg, Germany.

\section{Immunofluorescent assays}

Ten microlitres of each of the fluorescent MoAbs were added to $50 \mu \mathrm{l}$ heparinized blood. The mixture of blood and MoAbs was kept at room temperature in the dark for $15 \mathrm{~min}$. Then the erythrocytes were lysed with $2 \mathrm{ml}$ of erythrocyte lysis buffer (Becton Dickinson) for 15 min. After centrifugation at $200 \times \mathrm{g}$ for $5 \mathrm{~min}$, the pellet was washed twice with phosphate-buffered saline (PBS). Finally, the cells in the washed pellet were resuspended in $1 \%$ paraformaldehyde, and kept at $4^{\circ} \mathrm{C}$ until flow cytometric analysis.

BAL cells $\left(2 \times 10^{5}, 50 \mu \mathrm{l}\right)$ were incubated for $30 \mathrm{~min}$ at $4^{\circ} \mathrm{C}$ in the darkness, with $10 \mu \mathrm{l}$ of the indicated FITCand PE-MoAbs. After incubation, the cells were washed twice with PBS. The staining of the immunofluorescentlabelled cells was resuspended in $300 \mu \mathrm{l}$ of $1 \%$ paraformaldehyde in PBS. To distinguish between damaged and intact cells in fixed flow cytometric samples, the vital nuclei acid stain LDS 751 (Exciton, Dayton OH, USA) [14] was used.
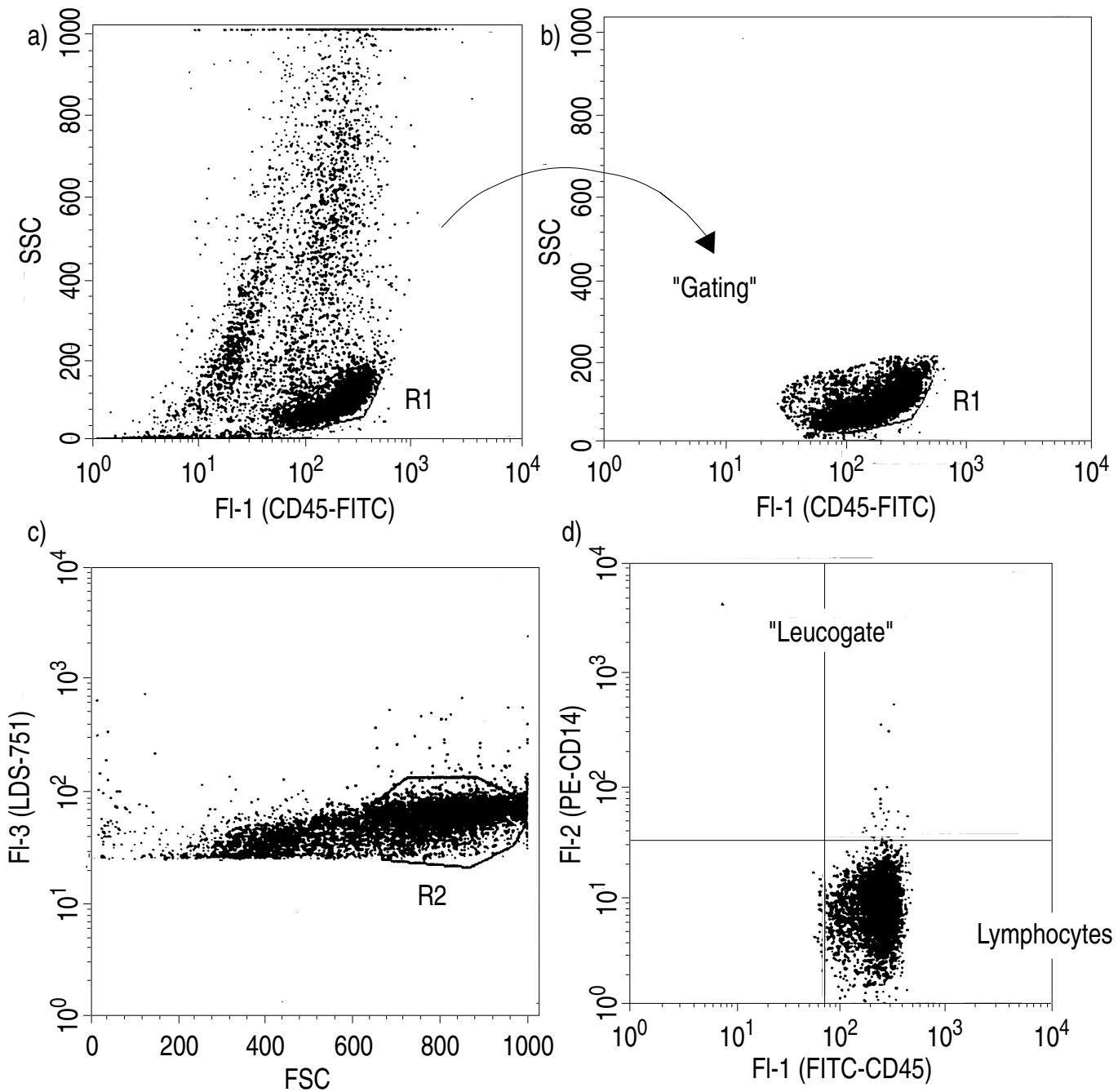

Fig. 1. - Flow cytometric analysis of bronchoalveolar lavage (BAL) lymphocytes in a representative sarcoidosis patient. Lymphocytes (R1) of the BAL were distinguished from macrophages and granulocytes by FITC-conjugated anti-CD45 (Fl-1) and side scatter plots (SSC) (Panels a and b). Intact lymphocytes (R2) were discriminated from damaged cells by forward light scatter (FSC) and LDS 751 stain (Fl-3) (Panel c). By a mixture of FITC-anti-CD45 (Fl-1) and PE-anti-CD14 (Fl-2), the purity of the lymphocyte gate (CD45+/side scatter) was controlled (Panel d). Panel $\mathrm{d}$ indicated that most of the cells $(>97 \%)$ in this gate were lymphocytes, because they were CD45-positive and CD14-negative. FITC: fluorescein isothiocyanate; PE: phycoerythrin. 
Flow cytometric analysis

A fluorescence-activated cell sorter system (FACSStar-Plus, Becton Dickinson) with an argon laser (488 $\mathrm{nm}$, air cooled) was used. Acquisition and analysis were carried out using the Lysis 1.0 programme. For each test, at least 10,000 cells were acquired.

Blood lymphocytes were discriminated from blood monocytes or neutrophils by characteristic forward light scatter (FSC) and right side light scatter (SSC) profiles. The characteristic scatter conditions were controlled by staining with a mixture of FITC-conjugated anti-CD45 and PE-conjugated anti-CD14 (leucogate). For twocolour immunfluorescence studies, the proportions of lymphocytes with positive staining for each antibody were calculated by setting four quadrants. Based on control samples, we divided the dot plots for unstained cells (lower left), and single stained cells: FITC-stained cells (lower right), PE-stained cells (upper left), and doublestained cells (upper right). Unstained cells are required to assess cellular autofluorescence. Directly conjugated subclass control cells (labelled with immunoglobulin G1 (IgG1)-FITC and (IgG2)-PE) will indicate nonspecific binding in direct staining experiments. Controls were subtracted, and the results were expressed as percentage of analysed gated cells.

BAL lymphocytes were distinguished from macrophages and granulocytes by fluorescence with FITCconjugated anti-CD45 and side scatter plots (SSC) (fig. $1)$. However, most of the cells $(>97 \%)$ in this fluorescence/side scatter gate are lymphocytes; this gate may also include small numbers of nonlymphoid cells (e.g. CD14-positive monocytes). To evaluate the number of these cells, they were analysed with an antibody mixture of FITC-conjugated anti-CD45 and PE-conjugated-anti CD14. Gated lymphocytes (by CD45+/side scatter gate) were FITC-anti-CD45 positive, and negative by PEanti-CD14 fluorescence (fig. 1d).

To detect T-cell subsets in the BAL, a mixture of FITCanti-CD45 and specific antibodies for the T-cell subsets conjugated with PE was used. For the identification of TCR bearing lymphocytes, fluorescence PE-conjugated anti-CD3, anti-CD4 or anti-CD8, and FITC-conjugated anti-TCR $\alpha / \beta$ or FITC-conjugated anti-TCR $\gamma / \delta$ were used.

\section{Statistical methods}

Quantitative data were expressed as the mean \pm standard deviation (SD). Wherever relevant, the Kruskal-Wallis test with a Monte Carlo estimation (by 100,000 tables) of p-values was used to compare the four and three groups of patients, respectively. To compare the data location of blood $\gamma / \delta \mathrm{T}$-lymphocytes between two patient groups, e.g. control and sarcoidosis, control and HP, control and IPF, or sarcoidosis and HP, the exact Wilcoxon rank sum test was used. Two-sided test p-values were calculated, and p-values of $<0.05$ were considered significant. Calculations were performed on a personal computer using StatXaxt statistical package (Cytel-Software, Corp., Cambridge, MA, USA).

\section{Results}

\section{Distribution of blood CD3+ $\gamma / \delta T C R$ cells}

Using two-colour direct immunofluorescence with the anti- $\gamma / \delta$ TCR and anti-CD3 MoAbs, flow cytometric analysis demonstrated that patients with pulmonary sarcoidosis and HP show a significant increase in the proportions of $\mathrm{CD} 3+\gamma / \delta+$ cells compared with normal subjects (fig. 2 ). Normal individuals had $3.2 \pm 1.4 \%$ (range $0.2-6.6 \%$ ) of blood T-cells that were CD3+ TCR $\gamma / \delta+$, and sarcoidosis patients had $8.3 \pm 7.9 \%$ (range $0.8-29.4 \%$ ). Using $7.4 \%$ (mean of the normal control group plus $3 \times \mathrm{SD}$ ) as the upper level of normals, a subgroup of 15 of the 31 $(48 \%)$ individuals with sarcoidosis had CD3 $+\gamma / \delta \mathrm{TCR}+$ lymphocytes above this level. Patients with HP had $5.8 \pm 3.5 \%$ (range $1.3-11.5$ ) of CD3+ $\gamma / \delta$ TCR lymphocytes in their blood, whereas IPF patients had $3.2 \pm 1.3 \%$ (range 0.9-7.4) (fig. 2). In the former group, five of the 13 subjects $(33 \%)$ expressed $\gamma / \delta$ TCR on more than $7.4 \%$ of their T-cells. There was no significant difference between HP and sarcoidosis patients in the proportion of CD3+ $\gamma / \delta \mathrm{TCR}+$ blood T-cells.

To determine the absolute number of CD3 $+\gamma / \delta+$ cells in the blood, data from the total white blood cell counts, cell differentials and the proportions of CD3+ $\gamma / \delta+$ cells measured by cytofluorometry were calculated. In sarcoidosis patients, $187.5 \pm 112.9$ cells $\cdot \mu \mathrm{l}^{-1}$ were $\mathrm{CD} 3+\gamma / \delta+$ TCR (mean \pm SD), whereas in normal subjects CD3 $+\gamma / \delta+$ cells were present in low numbers $\left(77.7 \pm 18.2\right.$ cells $\left.\cdot \mathrm{ul}^{-1}\right)$ (sarcoidosis versus control $\mathrm{p}<0.02$ ). In HP patients, $189.9 \pm 85.4$ cells $\cdot \mu l^{-1}$ were CD3+ $\gamma / \delta$ TCR (HP versus control $\mathrm{p}<0.01)$. In patients with IPF only $75.7 \pm 49.7{\text { cells } \cdot \mu{ }^{-}}^{-}$ ${ }^{1}$ had the $\gamma / \delta T C R$. These data indicate an increase in the total number of circulating blood CD $3+\gamma / \delta+$ cells in individuals with pulmonary sarcoidosis and HP.

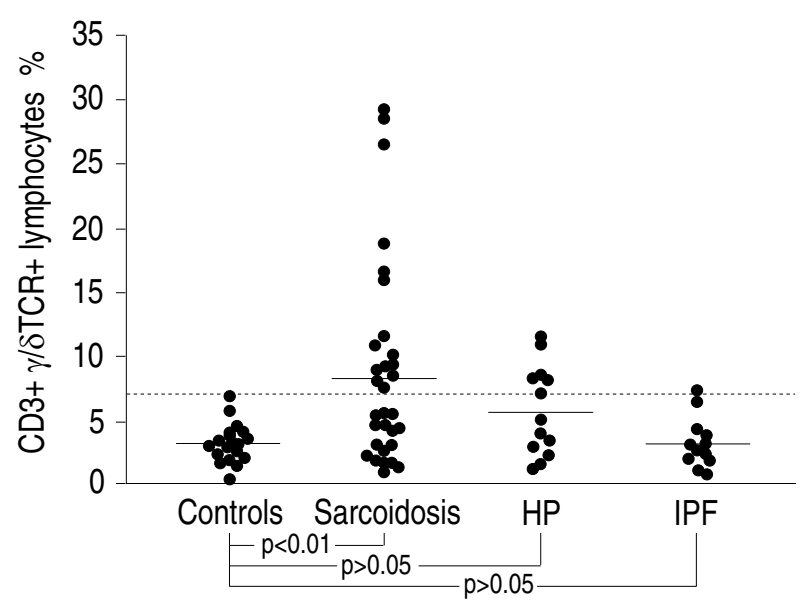

Fig. 2. - Proportion of CD $3+\gamma / \delta \mathrm{TCR}+$ lymphocytes in peripheral blood of healthy controls $(n=21)$, patients suffering from sarcoidosis $(n=31)$, hypersensitivity pneumonitis (HP) $(n=13)$ and idiopathic pulmonary fibrosis (IPF) $(n=12)$. Comparison of the four patient groups by Kruskal-Wallis test (Monte Carlo estimation) indicated that the four rows were different with $\mathrm{p}<0.02$. Indicated $\mathrm{p}$-values were formally calculated by the Wilcoxon rank sum test. — : mean value; $7.4 \%$, calculated value of upper normal value. TCR: T-cell receptor. 
Table 1. - Comparison of patients suffering from sarcoidosis with higher and lower levels of $\gamma / \delta$ TCRlymphocytes in blood

\begin{tabular}{|c|c|c|c|c|}
\hline & \multicolumn{2}{|c|}{$\begin{array}{l}\text { Patients with higher } \\
\text { CD3+ } \gamma / \delta \text { TCR level } \\
\quad(\geq 7.4 \%) n=15\end{array}$} & \multicolumn{2}{|c|}{$\begin{array}{c}\text { Patients with lower } \\
\text { CD3+ }+\gamma / \delta \text { TCR level } \\
(<7.4 \%) n=16\end{array}$} \\
\hline & $\mathrm{n}$ & $\%$ & $\mathrm{n}$ & $\%$ \\
\hline Female/Male & $9 / 6$ & $60 / 40$ & $11 / 5$ & $69 / 31$ \\
\hline $\mathrm{Age}^{\dagger}$ yrs & \multicolumn{2}{|c|}{$36 \pm 8.8$} & \multicolumn{2}{|c|}{$34 \pm 8.9$} \\
\hline Stage I & 4 & 27 & 7 & 44 \\
\hline Stage II & 11 & 73 & 9 & 56 \\
\hline Smokers & 3 & 20 & 3 & 19 \\
\hline Nonsmokers & 8 & 53 & 10 & 62 \\
\hline Exsmokers & 4 & 27 & 3 & 19 \\
\hline $\begin{array}{l}\text { Blood CD4/CD8 } \\
\text { ratio }\end{array}$ & \multicolumn{2}{|c|}{$0.74 \pm 0.36$} & \multicolumn{2}{|c|}{$1.17 \pm 0.59 *$} \\
\hline
\end{tabular}

†: mean \pm SD. *: $\mathrm{p}<0.05$. TCR: T-cell receptor.

Table 1 compares the data of sarcoidosis patients with increased $(\geq 7.4 \%)$ and normal proportions $(<7.4 \%)$ of blood CD3 $+\gamma / \delta+$ cells. With respect to age, sex and smoking behaviour, patients with increased numbers of CD3 $+\gamma / \delta$ TCR cells did not differ significantly from those with normal numbers of these T-cell subpopulations in the blood. Comparison of the blood CD4/CD8 ratios in these two groups demonstrated a significantly lower $\mathrm{CD} 4 / \mathrm{CD} 8$ ratio in the group of patients with higher $\mathrm{CD} 3+\gamma / \delta+$ levels $(\mathrm{p}<0.05)$. If the CD4/CD8 ratios were compared with the ratio of healthy controls $(1.49 \pm 0.52, \mathrm{n}=21)$, a significant difference existed only between sarcoidosis patients with higher $\mathrm{CD} 3+\gamma / \delta+$ levels and the control group $(\mathrm{p}<0.01)$. The $\mathrm{CD} 4 / \mathrm{CD} 8$ ratios of patients suffering from HP, both with normal and elevated CD $3+\gamma / \delta+$ levels in blood, and from IPF were in the normal range $(1.20 \pm 0.94$ and $1.25 \pm 1.09$ respectively).

\section{Correlation between $C D 4+$ and $C D 3+\gamma / \delta+$ lymphocytes in blood}

According to the differences between the CD4/CD8 ratio of control individuals and sarcoidosis patients, we compared the percentage of CD4+ lymphocytes in blood of healthy subjects with that of sarcoidosis patients. The data indicated a significant decrease of CD4+ lymphocytes in the blood of sarcoidosis patients $(n=31)$ versus normal volunteers $(\mathrm{n}=21)(26 \pm 10.9$ versus $42 \pm 6.6$; $\mathrm{p}<0.01$ ) (fig. 3). (13 out of 15) sarcoidosis patients who expressed $\gamma / \delta$ TCR on more than $7.4 \%$ of their lymphocytes had less than $30 \%$ of CD4+ lymphocytes (impoverishment of CD4 in blood). In all normal individuals examined, the percentage of CD4+ blood lymphocytes was higher than $30 \%$.

The percentages of CD4+ lymphocytes in patients with IPF and HP did not differ from normal values $(36 \pm 11.0 \%$ in IPF and $37 \pm 11.8 \%$ in HP). Only two HP patients expressing $\gamma / \delta+$ TCR on more than $7.4 \%$ of their lymphocytes had a CD4+ percentage below $30 \%$.

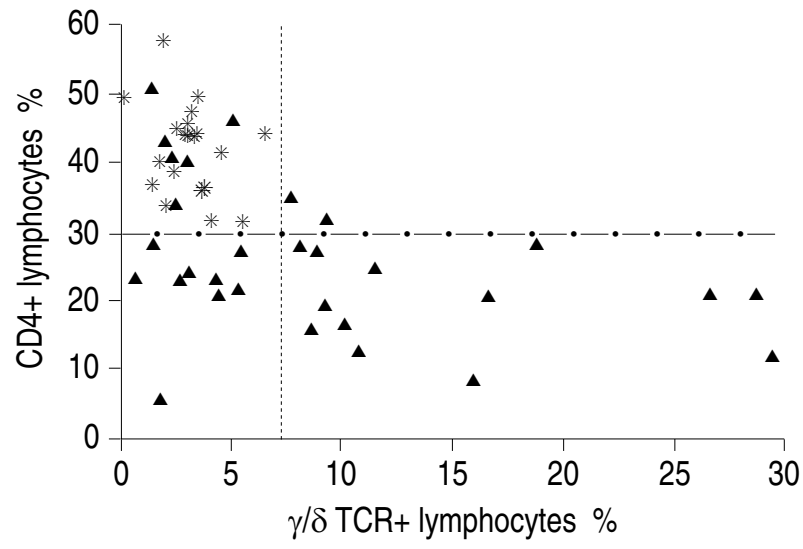

Fig. 3. - Comparison of the percentage of CD4+ lymphocytes and $\gamma / \delta$ TCR + lymphocytes in the blood of healthy subjects and patients suffering from pulmonary sarcoidosis. Data indicate that the percentage of CD4+ lymphocytes in all normal individuals was higher than $30 \%$ (the $30 \%$ range is marked by the line - -- ) and that sarcoidosis patients (13 out of 15 ) who expressed $\gamma / \delta \mathrm{TCR}$ on more than $7.4 \%$ of their lymphocytes (marked by line ......) had less than $30 \%$ of CD4+ lymphocytes. $\mathbf{\Delta}$ : sarcoidosis $(n=31)$; $*$ : controls $(n=21)$.

Association between $\gamma / \delta+T C R$ of blood T-lymphocytes and accessory molecules

Association between $\gamma / \delta+$ TCR of blood T-lymphocytes and the accessory molecules CD4 or CD8 was performed in blood of sarcoidosis and in healthy controls. The data on seven patients indicated that $46 \pm 13.9 \%$ of $\gamma / \delta \mathrm{TCR}+$ T-cells were CD8-positive, and $50 \pm 15.1 \%$ of the $\gamma / \delta+$ cells were neither CD8 nor CD4, having a "double negative phenotype". The number of cells scored as $\gamma / \delta+$ CD4+ $(3.5 \pm 2.8 \%)$ was equivalent to the background levels of staining, indicating that $\gamma / \delta+\mathrm{TCR}$ lymphocytes were CD4-negative. The association between the $\gamma / \delta+\mathrm{TCR}$ expression and the phenotypes CD4-CD8+ or CD4-CD8were irrespective of whether the sarcoidosis patients had elevated or normal percentages of circulating $\gamma / \delta+\mathrm{TCR}$ lymphocytes. Concerning this association, no significant difference existed between sarcoidosis patients and healthy controls; in the last-mentioned group $38 \pm 4.1 \%$ of $\gamma / \delta \mathrm{TCR}+$ cells also bear CD8, and $62 \pm 3.1 \%$ had a "double-negative" phenotype (CD4-CD8-).

To determine whether $\gamma / \delta$ TCR+ lymphocytes had been recently activated, the expression of the IL-2 receptor (CD25) was evaluated. Double staining experiments with PE-conjugated anti-CD25 and FITC-anti- $\gamma / \delta$ TCR from all tested individuals (eight sarcoidosis patients and four healthy controls) demonstrated that the $\gamma / \delta \mathrm{TCR}+$ lymphocytes are CD25-negative $(0.4 \pm 0.5 \%, \mathrm{n}=12)$.

\section{Distribution of $C D 3+\gamma / \delta+T$-cells in $B A L$}

The evaluation of lymphocytes present in the BAL indicated a higher percentage of CD $3+\gamma / \delta \mathrm{TCR}+$ cells in HP patients than in IPF, $\mathrm{p}<0.05$ (fig. 4 , table 2). The percentage of $\mathrm{CD} 3+\gamma / \delta \mathrm{TCR}+$ of patients with sarcoidosis was not significantly different from that of patients with HP or IPF. 


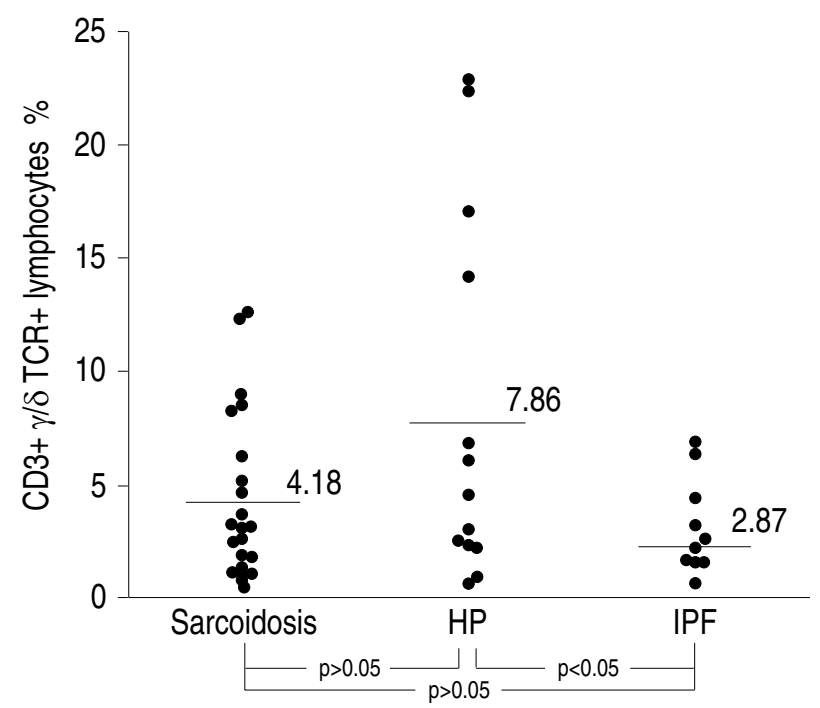

Fig. 4. - Proportion of $\gamma / \delta T C R$ expression of lymphocytes in the bronchoalveolar lavage in patients suffering from sarcoidosis $(n=20)$, hypersensitivity pneumonitis (HP) $(\mathrm{n}=13)$ and idiopathic pulmonary fibrosis (IPF) $(n=12)$. Indicated p-values were formally calculated by the exact Wilcoxon rank sum test. ——: mean. TCR: T-cell receptor.

Compared to IPF in the BAL of patients suffering from sarcoidosis and HP the absolute number and the percentage values of lymphocytes were significantly higher.
In contrast to percentage values, the total amounts of $\mathrm{CD} 3+\gamma / \delta \mathrm{TCR}+($ table 2$)$ showed significant differences in sarcoidosis, HP and IPF (sarcoidosis versus IPF, $\mathrm{p}<0.02$; HP versus IPF, $\mathrm{p}<0.05$ and sarcoidosis versus $\mathrm{HP}, \mathrm{p}<0.05)$.

The 20 sarcoidosis patients had $4.2 \pm 3.6 \%$ (range $0.8-12.8 \%)$ of CD3 $+\gamma / \delta$ TCR + cells. Ten of these sarcoidosis patients belonged to the subgroup with increased blood levels of CD $3+\gamma / \delta \mathrm{TCR}+(\geq 7.4 \%$ of CD $3+\gamma / \delta \mathrm{TCR}+$ cells). In addition, the cellular distribution of the BAL fluids of the two sarcoidosis subgroups, with increased and normal blood levels of CD3+ $\gamma / \delta \mathrm{TCR}+$, was compared (table 3). Sarcoidosis patients with higher blood CD3 $+\gamma / \delta$ TCR + levels showed significantly decreased total cell numbers $\cdot \mathrm{ml}^{-1} \mathrm{BAL}$ and lymphocytes $\cdot \mathrm{ml}^{-1}(\mathrm{p}<0.05)$, compared with the other sarcoidosis subgroup. Corresponding with the higher percentage of blood CD3+ $\gamma / \delta \mathrm{TCR}+$ levels, this subgroup also had an increased percentage of $\mathrm{CD} 3+\gamma / \delta+$ in the BAL $(\mathrm{p}<0.05)$, but the total number of $\mathrm{CD} 3+\gamma / \delta \mathrm{TCR}+$ cells $\cdot \mathrm{ml}^{-1}$ was not different in the two patient groups. Calculating the BAL CD4/CD8 ratios of the two groups, no significant difference was observed.

Comparison of BAL data obtained from HP patients with increased (5 out of 13 patients) and normal (8 out of 13) blood CD3+ $\gamma / \delta+$ levels demonstrated no significant difference with respect to BAL CD3 $+\gamma / \delta+$ levels $(7.7 \pm 8.2(\mathrm{n}=5)$ versus $9.3 \pm 9.07(\mathrm{n}=8) ; \mathrm{p}>0.05)$ and CD4/CD8 ratios $(0.78 \pm 0.54(\mathrm{n}=5)$ versus $0.63 \pm 0.31(\mathrm{n}=8)$; $\mathrm{p}>0.05)$.

Table 2. - Lymphocytes and CD3+ $\gamma / \delta$ TCR expression (percentage and absolute number) in BAL of patients suffering from sarcoidosis, hypersensitivity pneumonitis (HP) or idiopathic pulmonary fibrosis (IPF)

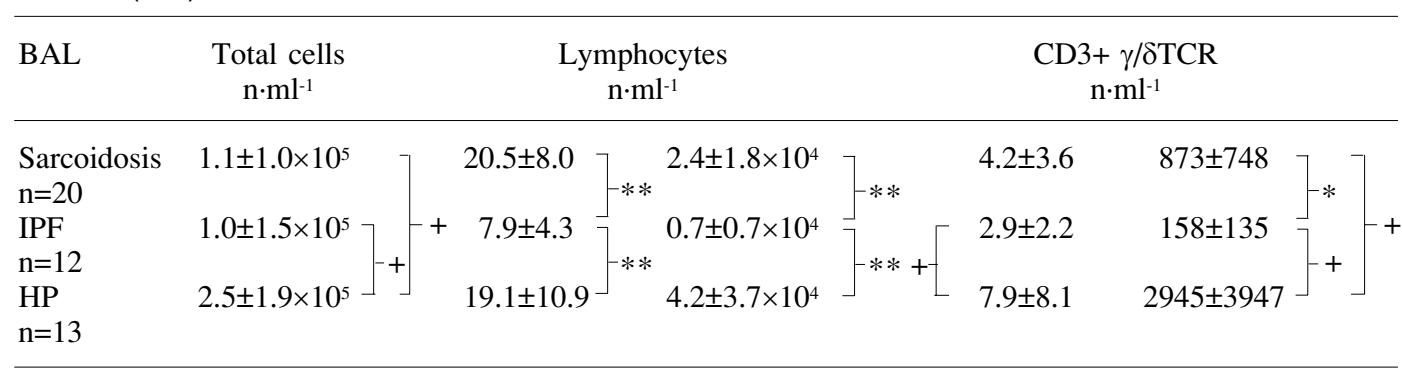

Data are presented as mean \pm SD. +: $\mathrm{p}<0.05 ; *$ : $\mathrm{p}<0.02 ; * *: \mathrm{p}<0.01$. BAL: bronchoalveolar lavage; TCR: T-cell receptor.

Table 3. - Comparison of BAL fluid in sarcoidosis patients with higher and normal blood CD3+ $\gamma / \delta$ TCR levels

\begin{tabular}{|c|c|c|c|}
\hline & $\begin{array}{l}\text { Patients with higher } \\
\text { blood CD3+ } \gamma / \delta \mathrm{TCR} \\
\text { level }(\geq 7.4 \%) \mathrm{n}=10\end{array}$ & $\begin{array}{c}\text { Patients with lower } \\
\text { blood CD3+ } \gamma / \delta \text { TCR } \\
\text { level }(<7.4 \%) n=10\end{array}$ & $\mathrm{p}$ \\
\hline BAL total cells & $0.6 \pm 0.5 \times 10^{5}$ & $1.4 \pm 1.2 \times 10^{5}$ & $<0.05$ \\
\hline Lymphocytes \% & $18 \pm 7.3$ & $22 \pm 8.0$ & NS \\
\hline $\mathrm{n} \cdot \mathrm{ml}^{-1}$ & $1.6 \pm 1.1 \times 10^{4}$ & $3.2 \pm 2.1 \times 10^{4}$ & $<0.05$ \\
\hline $\mathrm{CD} 3+\gamma / \delta \mathrm{TCR}+\%$ & $4.8 \pm 2.2$ & $1.8 \pm 0.9$ & $<0.05$ \\
\hline $\mathrm{n} \cdot \mathrm{ml}^{-1}$ & $939.6 \pm 936.4$ & $782.0 \pm 679$ & NS \\
\hline CD4/CD8 ratio & $8.7 \pm 6.3$ & $5.6 \pm 6.1$ & NS \\
\hline
\end{tabular}

Data are presented as mean \pm SD. NS: nonsignificant. For further abbreviations see legend to table 2. 


\section{Discussion}

Our data demonstrate elevated levels of T-cells bearing the $\mathrm{CD} 3+\gamma / \delta \mathrm{TCR}$ in blood of patients suffering from sarcoidosis and HP. In both patient groups, this expansion of $\gamma / \delta \mathrm{T}$-cells was clearly defined and significantly different from blood of controls and patients suffering from IPF, when considering both the mean value of the percentage or the total numbers of CD $3+\gamma / \delta \mathrm{TCR}+$ cells. However, the proportions of $\gamma / \delta \mathrm{TCR}+\mathrm{T}$-lymphocytes in blood of sarcoidosis and HP patients were quite variable, and ranged from $1.0-29 \%$, and more than $50 \%$ of individuals in both patient groups had circulating $\gamma / \delta \mathrm{TCR}+$ T-lymphocytes in the normal range.

Our data on $\gamma / \delta$ TCR expression in sarcoidosis patients are in agreement with those of BALBI et al. [6], who found increased numbers of $\alpha / \beta$-TCR cells in the blood of a subgroup of individuals with sarcoidosis. Our study confirmed that $\mathrm{CD} 3+\alpha / \beta-\mathrm{T}$-cells were actually $\mathrm{CD} 3+$ $\gamma / \delta+$ T-cells. In contrast to these data and ours, TAZI et al. [15] did not find an increase of the absolute number of CD3 $+\gamma / \delta \mathrm{T}$-cells in patients with sarcoidosis. They suggested that an increased proportion of circulating $\mathrm{CD} 3+\gamma / \delta \mathrm{T}$-cells resulted from a reduction in the number of lymphocytes bearing the $\alpha / \beta T C R$. Our results clearly demonstrate that the increase in $\gamma / \delta \mathrm{T}$-cells was due to an increase in the absolute number of blood $\gamma / \delta \mathrm{T}$ cells, but it must be considered that this phenomenon may differ in various study populations of sarcoidosis. Furthermore, TAZI et al. [15] demonstrated that the $\gamma / \delta \mathrm{T}$ cells in the blood play a limited role in the ongoing immune response in established tuberculosis. These results are strikingly different compared to the important increase in number of $\gamma / \delta \mathrm{T}$-lymphocytes observed in the lung and lymph nodes of mice exposed to Mycobacterium tuberculosis [16]. In addition, FORRESTER et al. [17] described that a subgroup of sarcoidosis patient, 6 out of $11,(54 \%)$ had increased $\gamma / \delta \mathrm{T}$-cells in blood, whereas in patients with chronic beryllium disease only 2 out of 9 individuals $(22 \%)$ had elevated blood $\gamma / \delta \mathrm{T}$-cells.

To understand why certain patients suffering from sarcoidosis have increased numbers of $\gamma / \delta \mathrm{T}$-cells, we compared some personal and clinical data of the patient groups with higher and normal levels of $\gamma / \delta T C R$. Concerning age, sex, smoking behaviour and sarcoidosis stage, no significant difference existed between the two groups. However, a significantly lower CD4/CD8 ratio was demonstrated in the group of patients with higher $\gamma / \delta$ TCR levels. In addition, our data clearly demonstrate a good correlation between the decrease of CD4+ blood cells and the amplified appearance of $\gamma / \delta$ TCR expression in patients with sarcoidosis. It seemed possible, that in sarcoidosis the expansion of blood $\gamma / \delta \mathrm{T}$-cells was due to an increase of CD8, corresponding to the decrease of CD4 cells. In contrast, in HP patients the specific expansion of $\gamma / \delta \mathrm{T}$-cells seems to be independently regulated, irrespectively of a CD4 decrease. The clear correlation between CD4+ decrease and $\gamma / \delta \mathrm{TCR}+$ increase may be of particular interest in sarcoidosis patients.

Moreover, our study indicates that the $\gamma / \delta$ TCR of blood T-lymphocytes was associated either with CD8 in approxi- mately $50 \%$ or with a "double negative phenotype" (CD4CD8-) on the remaining $50 \%$ of the $\gamma / \delta$ T-cells. Similar to our results, which demonstrate that only $3.5 \pm 2.8 \%$ of the $\gamma / \delta$ T-cells bear CD4, INGHIRAMI et al. [18] reported that all $\gamma / \delta$ TCR bearing peripheral lymphocytes lack detectable CD4, whilst approximately $60 \%$ of the $\gamma / \delta$ TCR thymocytes express low density CD4. Although CD4 expression on peripheral blood $\gamma / \delta$-cells is even less common than CD8 expression, CD4 $\gamma / \delta$ T-cells had been identified and cloned [19]. An further study [20] showed that approximately $30 \%$ of the $\gamma / \delta$-cells in the blood were CD8+, and BuCY et al. [20] and INGHIRAMI et al. [18] concluded that $\gamma / \delta \mathrm{T}$-cells are resting, mature T-cells that probably play a primary role in suppressor/cytotoxic phenomena and recognize antigens in association with classical or non-classical class I MHC molecules. Because all thymic $\gamma / \delta \mathrm{T}$-cells are $\mathrm{CD} 8+$, it will be important in this context to determine whether CD8 expression by $\gamma / \delta \mathrm{T}$-cells represents a normal stage of maturation, or occurs only after cell activation.

Finally, our data indicate, irrespective of elevated or normal levels of circulating $\gamma / \delta \mathrm{T}$-cells, that these cells were IL-2 receptor-negative (CD25-/ $/ / \delta \mathrm{TCR}+)$, suggesting a lack of activation. Interestingly, looking for the expression of other cell surface antigens, e.g. CD45RO as a marker of memory cells, it seemed that $\gamma / \delta \mathrm{T}$-cells have been preactivated in vivo [21]. It has to be considered that certain activation signals on $\alpha / \beta$-T-cells may have opposite effects on $\gamma / \delta$ T-cells [22], and that an activation of $\gamma / \delta \mathrm{T}$-cells does not correspond to the expression of usual activation markers, such as CD25.

In addition, our data demonstrate an increase of $\gamma / \delta \mathrm{TCR}+$ cells (absolute numbers) in BAL of patients suffering from HP and sarcoidosis, compared to BAL of IPF patients. In the subgroup of sarcoidosis patients with higher percentages of $\mathrm{CD} 3+\gamma / \delta \mathrm{TCR}+$ level, an increased percentage of $\mathrm{CD} 3+\gamma / \delta \mathrm{TCR}+$ cells in BAL was also observed. These data suggest that the $\gamma / \delta \mathrm{T}$-cells were not restricted to one compartment, because a corresponding increase in blood and lung (BAL) was seen in sarcoidosis patients.

In agreement with our results, TRENTIN et al. [23] found that the absolute number of $\gamma / \delta$ TCR-bearing T-cells was increased in the BAL of patients suffering from farmers' lung disease. Our study also demonstrated an increase of CD3+ $\gamma / \delta$ T-cells in the blood and BAL of patients with HP caused by other antigens. In contrast to sarcoidosis patients, the comparison between blood and BAL CD3+ $\gamma / \delta$ TCR+ levels of HP patients does not show a clear correlation.

Our study suggests that this CD $3+\gamma / \delta$ TCR-lymphocyte subpopulation might be of pathogenetic relevance in the blood and lung of patients with sarcoidosis and HP. These are two different interstitial lung disorders, with specific pathogenetic mechanisms and characteristics. SEMENZATo [24] concluded that the mechanisms accounting for alveolitis in sarcoidosis are an in situ cellular proliferation and a cellular redistribution from the peripheral blood to the site of the disease activity (i.e. the lung). Redistributed cells are especially the CD4+-helper related cells. In patients with HP, the BALs are characterized by 
cells bearing suppressor/cytotoxic phenotypes. The expansion of cells with the mentioned characteristics in the BAL of these patients is likely to be related to a local immune response to the antigenic stimulus. It has also to be considered that, in contrast to sarcoidosis, the BAL of patients with HP contains CD8+ cells as the dominant lymphocyte subpopulation. However, the absolute number of $\gamma / \delta+\mathrm{T}$-cells in BAL from HP patients is not higher than in BAL from sarcoidosis patients.

In conclusion, our data demonstrate elevated levels of $\gamma / \delta \mathrm{T}$-cells in sarcoidosis and HP patients suggesting a pathogenetic role of $\gamma / \delta \mathrm{TCR}$-bearing T-cells. It has not yet been clarified whether the bias in CD3+ $\gamma / \delta$ TCR expression is a causal factor for the development of such a disease, or whether it is a consequence of it.

Acknowledgements: The authors would like to thank G. Borowitzki, C. Krekel and A. Urbanski for their technical assistance and P. Degens for statistical assistance.

\section{References}

1. Hermann A, Kappler JW, Marrack P, Puller AM. Super-antigens: mechanisms of T-cell stimulation and role in immune response. Ann Rev Immunol 1991; 9: 745-772.

2. Raulet DH. - The structure, function and molecular genetics of the $\gamma / \delta$ T-cell receptor. Ann Rev Immunol 1989; 7: 175-207.

3. Holoshitz J, Vila LM, Keroack BJ, McKinley DR, Bayne NK. - Dual antigenic recognition by cloned human

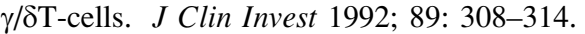

4. Thomas PD, Hunninghake GW. - Current concepts of pathogenesis of sarcoidosis. Am Rev Respir Dis 1987; 135: 747-760.

5. Tamura N, Moller DR, Balbi B, Crystal RG. - Preferential usage of the T-cell antigen receptor $\beta$-chain constant region $\mathrm{C} \beta 1$ element by lung T-lymphocytes of patients with pulmonary sarcoidosis. Am Rev Respir Dis 1991; 143: 635-639.

6. Balbi B, Moller DR, Kirby M, Holrody KJ, Crystal RG. - Increased numbers of T-lymphocytes with $\gamma / \delta$ positive antigen receptors in a subgroup of individuals with pulmonary sarcoidosis. J Clin Invest 1990; 85: $1353-1361$.

7. Salvaggio JE. - Recent advances in pathogensis of allergic alveolitis. Clin Exp Allergy 1990; 20: 137-144.

8. Richerson HB, Bernstein IL, Fink JN. - Guidelines for the clinical evaluation of hypersensitivity pneumonitis: report of the subcommittee on hypersensitivity pneumonitis. J Allergy Clin Immunol 1989; 84: 839-844.

9. Costabel U, Bross KJ, Marxen J, Matthy H. - Tlymphocytes in bronchoalveolar lavage fluid of hyper- sensititvity pneumonitis: changes in profile of T-cell subsets during the course of disease. Chest 1984; 85 : 514-519.

10. Stankus RP, Cashner FM, Salvaggio JE. - Bronchopulmonary macrophage activation in the pathogenesis of hypersensitivity pneumonitis. J Immunol 1978; 120: 685-688.

11. Haslam PL, Dewar A, Butchers P, Primett ZS, NewmanTaylor A, Turner-Warwick M. - Mast cells, atypical lymphocytes, and neutrophils in bronchoalveolar lavage in extrinsic allergic alveolitis. Am Rev Respir Dis 1987; 135: 35-47.

12. Hauk R, Baur X. - Verlaufsformen der Befeuchterlunge. Klin Wochenschr 1990; 68: 512-517.

13. Baur X, Römmelt H, Hoffarth HP, Fruhmann G. Exogen-allergische Alveolitis durch Isocyanate. Klin Wochenschr 1991; 69 (Suppl. XXIII): 297-298.

14. Terstappen LWMM, Loken MR. - Five-dimensional flow cytometry as a new approach for blood and bone marrow differentials. Cytometry 1988; 9: 548-556.

15. Tazi A, Bouchonnet F, Valeyre D, Cadrandel J, Battesti JJ, Hance AJ. - Characterization of $\gamma / \delta$ T-lymphocytes in peripheral blood of patients with active tuberculosis. Am Rev Respir Dis 1992: 146: 1216-1221.

16. Augustin AD, Kubo RT, Sim GK. - Resident pulmonary lymphocytes expressing the $\gamma / \delta \mathrm{T}$-cell receptor. Nature 1989; 340: 239-241.

17. Forrester JM, Newman LS, King Jr TE, Kotzin BL. Increased $\gamma / \delta \mathrm{T}$-cell receptor expression in blood but not bronchoalveolar lavage (BAL) fluid in patients with sarcoidosis and chronic beryllium disease. Am Rev Respir Dis 1991: 143: A219.

18. Inghirami G, Zhu BY, Chess L, Knowles DM. - Flow cytometric and immunhistochemical characterization of the $\gamma / \delta \mathrm{T}$-lymphocyte population in normal human lymphoid tissue and peripheral blood. Am J Pathol 1990; 136: 357-367.

19. Spits H, Paliard X, Vandekerckhove Y, van Vlasselaer P, De Vries J. - Functional and phenotypic diferences between CD4+ and CD4- T-cell receptor- $\gamma / \delta$ clones from peripheral blood. J Immunol 1991; 147: 1180-1186.

20. Bucy RP, Chen Ch-L, Cooper MD. - Tissue localization and CD8 accessory molecule expression of T $\gamma / \delta$ cells in humans. J Immunol 1989; 142: 3045-3049.

21. Miyawaki T, Kasahara Y, Taga K, Yachie A, Taniguchi N. - Differential expression of CD45RO (UCHL1) and its functional relevance in two subpopulations of circulating TCR- $\gamma / \delta+$ lymphocytes. J Exp Med 1990; 171: 1833-1839.

22. Kabelitz D. - Function and specificity of human $\gamma / \delta$ positive T-cells. Crit Rev Immumol 1992; 11: 281-303.

23. Trentin L, Migone N, Zambello R, et al. - Mechanisms accounting for lymphocytic alveolitis in hypersensitivity pneumonitis. J Immunol 1990; 145: 2147-2154.

24. Semenzato G. - Immunology of interstitial lung diseases: cellular events taking place in the lung of sarcoidosis, hypersensitity pneumonitis and HIV infection. Eur Respir J 1991; 4: 94-102. 